\title{
Preface of the Special JOGO issue in Memory of Professor Christodoulos A. Floudas (1959-2016)
}

\author{
Ignacio Grossmann ${ }^{1} \cdot$ Panos Pardalos ${ }^{2}$
}

Published online: 3 July 2018

(c) Springer Science+Business Media, LLC, part of Springer Nature 2018

Almost 2 years have passed since our great friend and colleague, Professor Christodoulos (Chris) Floudas, passed away at the age of 56. This special issue is dedicated to the memory of Professor Chris Floudas and his legacy.

Professor Floudas was an internationally highly esteemed researcher and his superb academic reputation crossed the boundaries of Chemical Engineering, Optimization, Applied Mathematics and Operations Research, and Computational Chemistry and Biology. In Chemical Engineering, this is attributed to his fundamental contributions to the area of Process Systems Engineering with primary focus on Process Synthesis, and Design and Control of large-scale chemical processes. In Optimization, Applied Mathematics and Operations Research, this is attributed to his pioneering work in the areas of Global Optimization and Mixed-Integer Nonlinear Optimization.

This special issue consists of papers contributed from those who have had some interaction with Chris Floudas, including friends, students, and research collaborators. In the first contribution of this special issue, Stratos Pistikopoulos presents a short history of his academic journey and the numerous contributions of Chris to several areas of science and engineering. The topics of the remaining papers of the special issue are focused on theoretical aspects, algorithmic approaches and cover a wide spectrum of applications of important and interesting problem classes in global optimization.

We would like to take this opportunity to thank the Editor-in-Chief Sergiy Butenko for his constant reminders and encouragement, as well as the contributors and the referees for their efforts and cooperation in producing this special issue of the Journal of Global Optimization.

Chris Floudas' academic record is truly outstanding and places him at the very top of all researchers in his area. Furthermore, his scientific research has had a major impact across several disciplines. Chris will be immensely missed. May his memory be eternal!

June 2018

\footnotetext{
Panos Pardalos pardalos@ufl.edu

Ignacio Grossmann grossmann@cmu.edu

1 Pittsburgh, USA

2 Gainesville, USA
} 